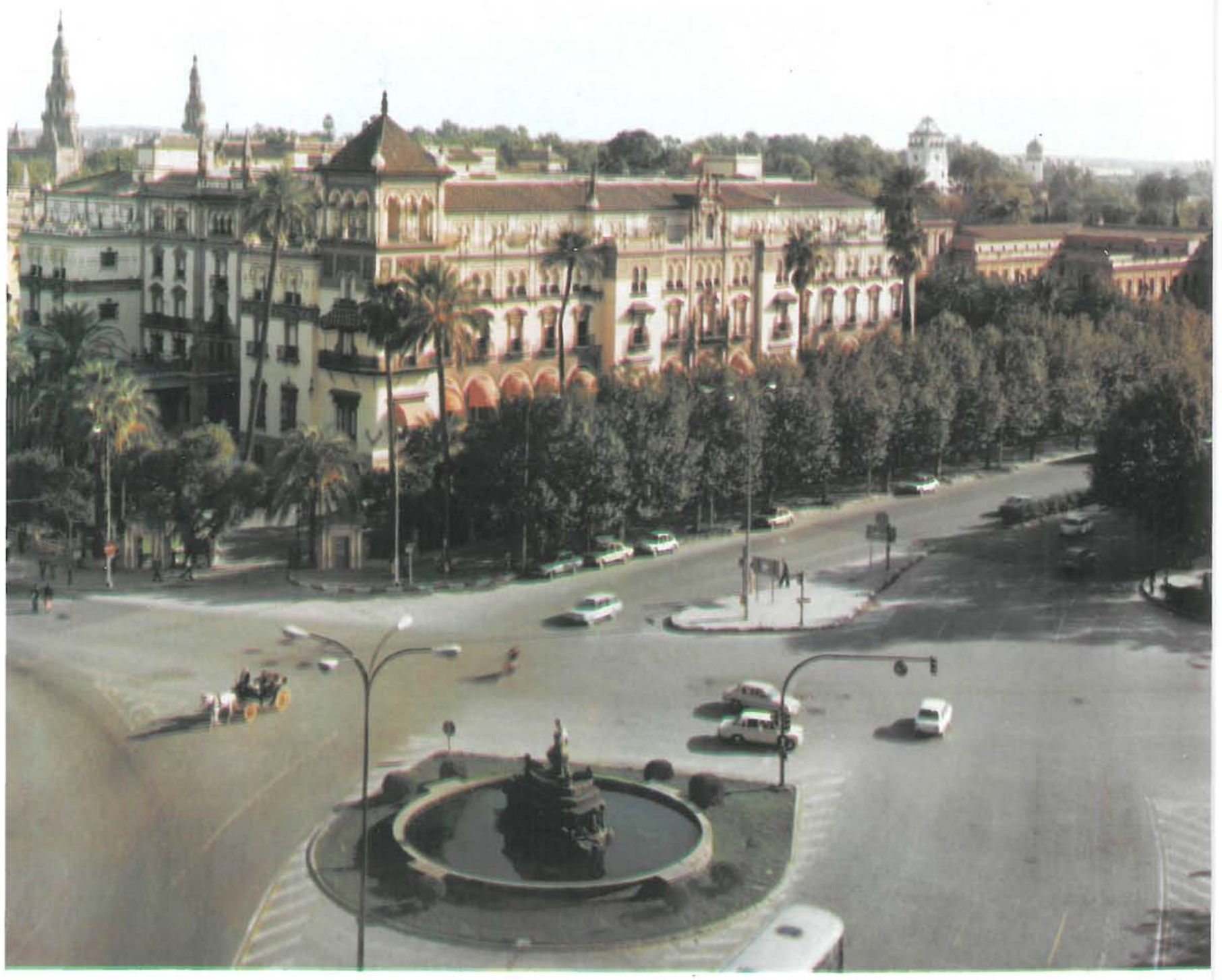

\title{
Reforma y modernización del Hotel Alfonso XIII de Sevilla
}

España

Carlos Picardo Castellón, Dr. arquitecto

$147-47$

\section{sinopsis}

Se describe en el articulo la cuidadosa reforma de este Hotel - parte de la Historia de la cludad, desde su Inauguración, en 1926-, con la consiguiente modernización general, al objeto de convertirlo en una instalación ejemplar, de gran belleza y dotada de todo el coniort que requiere su categoria de gran lujo, condición que comparte con muy pocos hoteles del Pais.

Se ha dedicado especial atención a la consolidación de la cublerta y tabiquería; renovación de la carpinteria exterior, verjas, azulejos, zócalos, artesonados, lámparas, forjado del comedor de banquetes, instalaciones de fontaneria, electricidad y aire acondiclonado; servicios adecuados, etcétera.

Se han creado: una magnifica y espaciosa sulte, baños de categoria, jardineria, estacionamientos cublertos, un aljibe de $800 \mathrm{~m}^{3}$, bar, cafeteria de desayunos, un restaurante en el semisótano - de uso exterior e interior-, fuentes...

El gusto esmerado del arquitecto, el trabajo altamente especializado de la empresa constructora y la aportación de la más depurada artesania sevillana, han contribuido a un perfecto acabado y a un resultado final de excepción. 

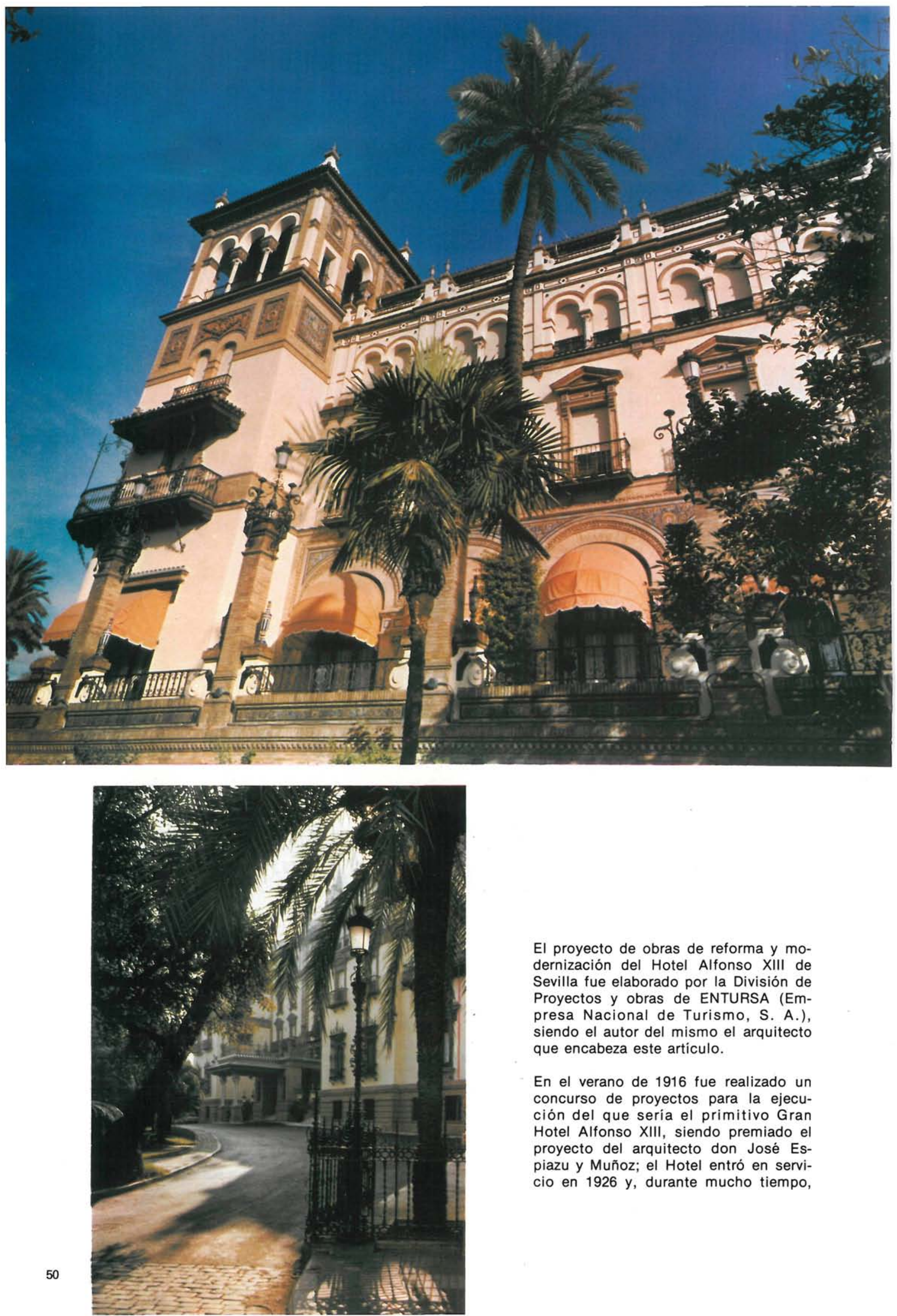

El proyecto de obras de reforma y modernización del Hotel Alfonso XIII de Sevilla fue elaborado por la División de Proyectos y obras de ENTURSA (Empresa Nacional de Turismo, S. A.), siendo el autor del mismo el arquitecto que encabeza este artículo.

En el verano de 1916 fue realizado un concurso de proyectos para la ejecución del que seria el primitivo Gran Hotel Alfonso XIII, siendo premiado el proyecto del arquitecto don José Espiazu y Muñoz; el Hotel entró en servicio en 1926 y, durante mucho tiempo, 


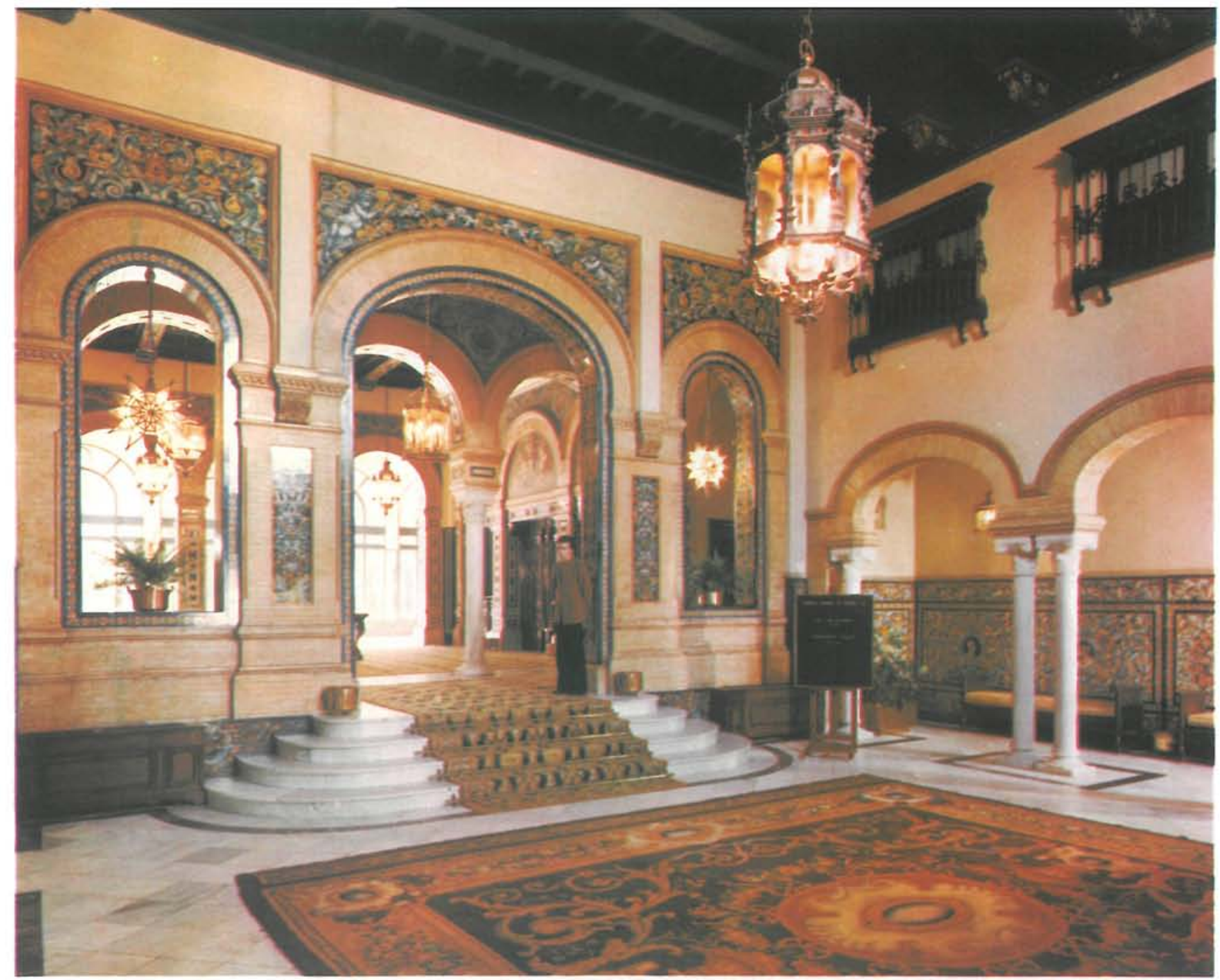

ofreció estancia en sus 160 habitaciones a los más ilustres visitantes de Sevilla.

Hace unos años, el Hotel hubo de ser cerrado por imperiosas necesidades de reforma y puesta al dia; y ahora, de acuerdo con este nuevo Proyecto, han sido acondicionadas todas sus dependencias y habitaciones con bastantes modificaciones en orden a un mejor $y$ más moderno servicio, consiguiendo mantener toda su original grandeza y suntuosidad, recuperando asi Sevilla la pervivencia de un edificio entrañable que es ya parte de su historia.

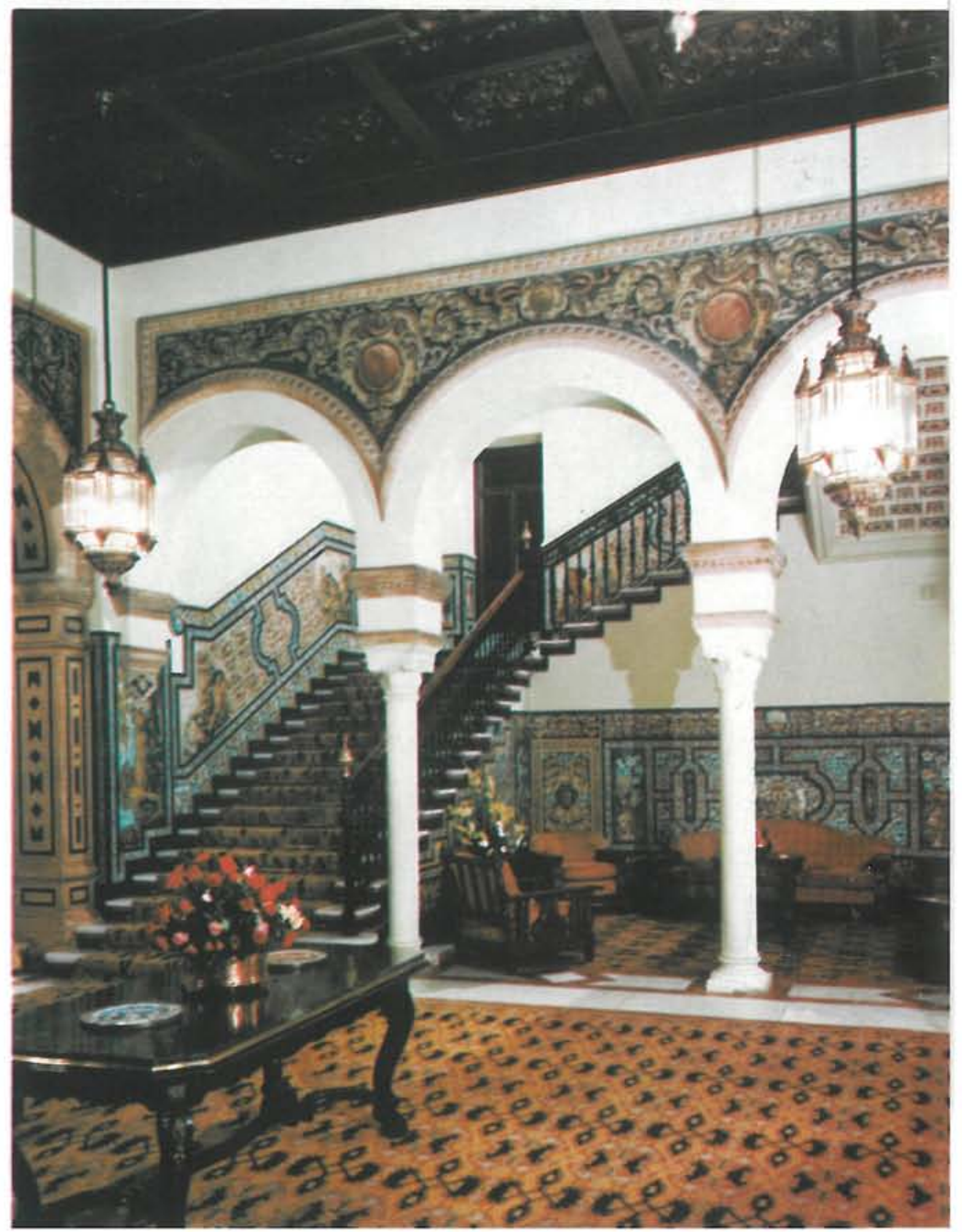




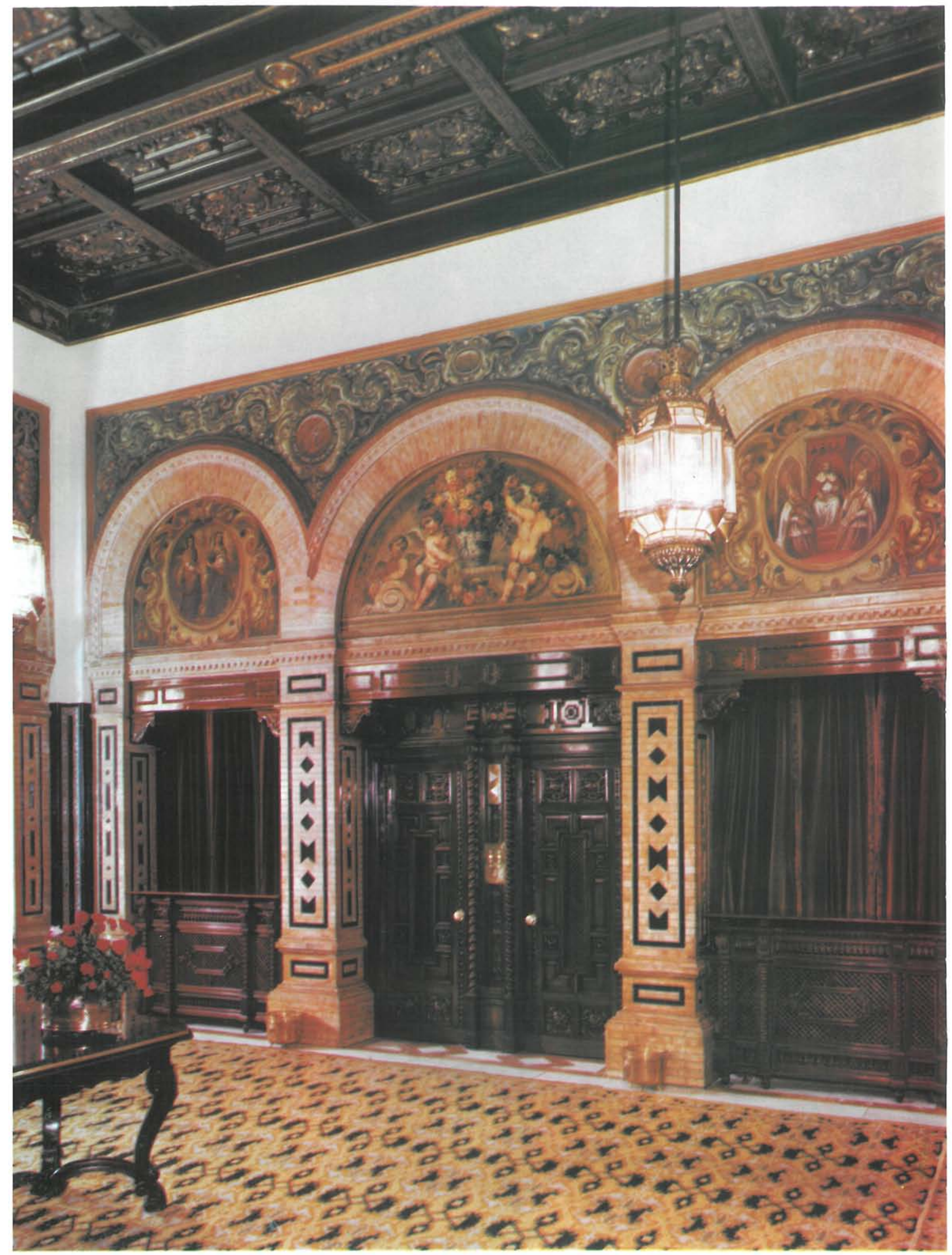

52 

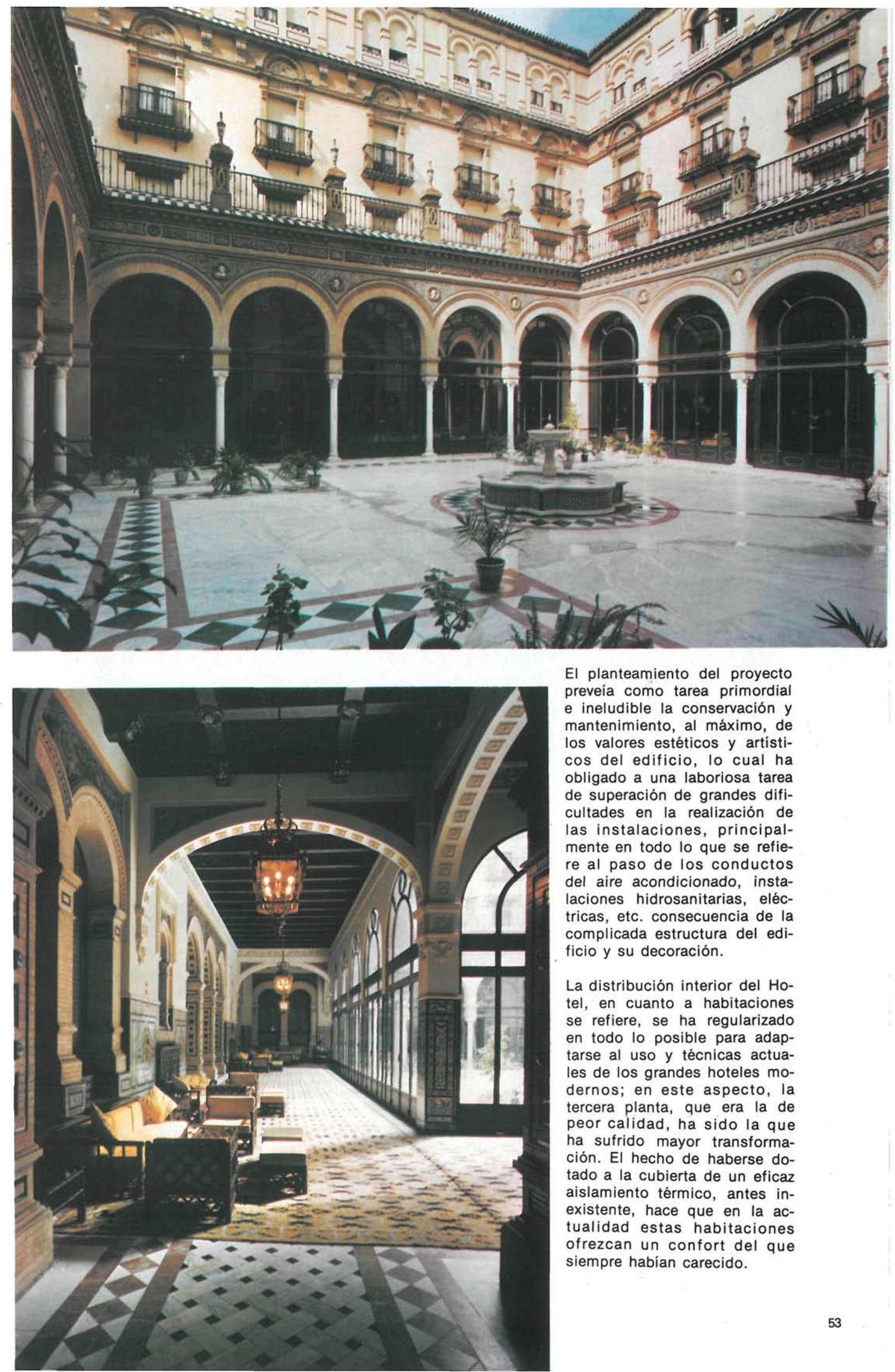

El planteamiento del proyecto preveia como tarea primordial e ineludible la conservación y mantenimiento, al máximo, de los valores estéticos y artisticos del edificio, lo cual ha obligado a una laboriosa tarea de superación de grandes dificultades en la realización de las instalaciones, principalmente en todo lo que se refiere al paso de los conductos del aire acondicionado, instalaciones hidrosanitarias, eléctricas, etc. consecuencia de la complicada estructura del edificio y su decoración.

La distribución interior del Hotel, en cuanto a habitaciones se refiere, se ha regularizado en todo lo posible para adaptarse al uso y técnicas actuales de los grandes hoteles modernos; en este aspecto, la tercera planta, que era la de peor calidad, ha sido la que ha sufrido mayor transformación. El hecho de haberse dotado a la cubierta de un eficaz aislamiento térmico, antes inexistente, hace que en la actualidad estas habitaciones ofrezcan un confort del que siempre habian carecido. 


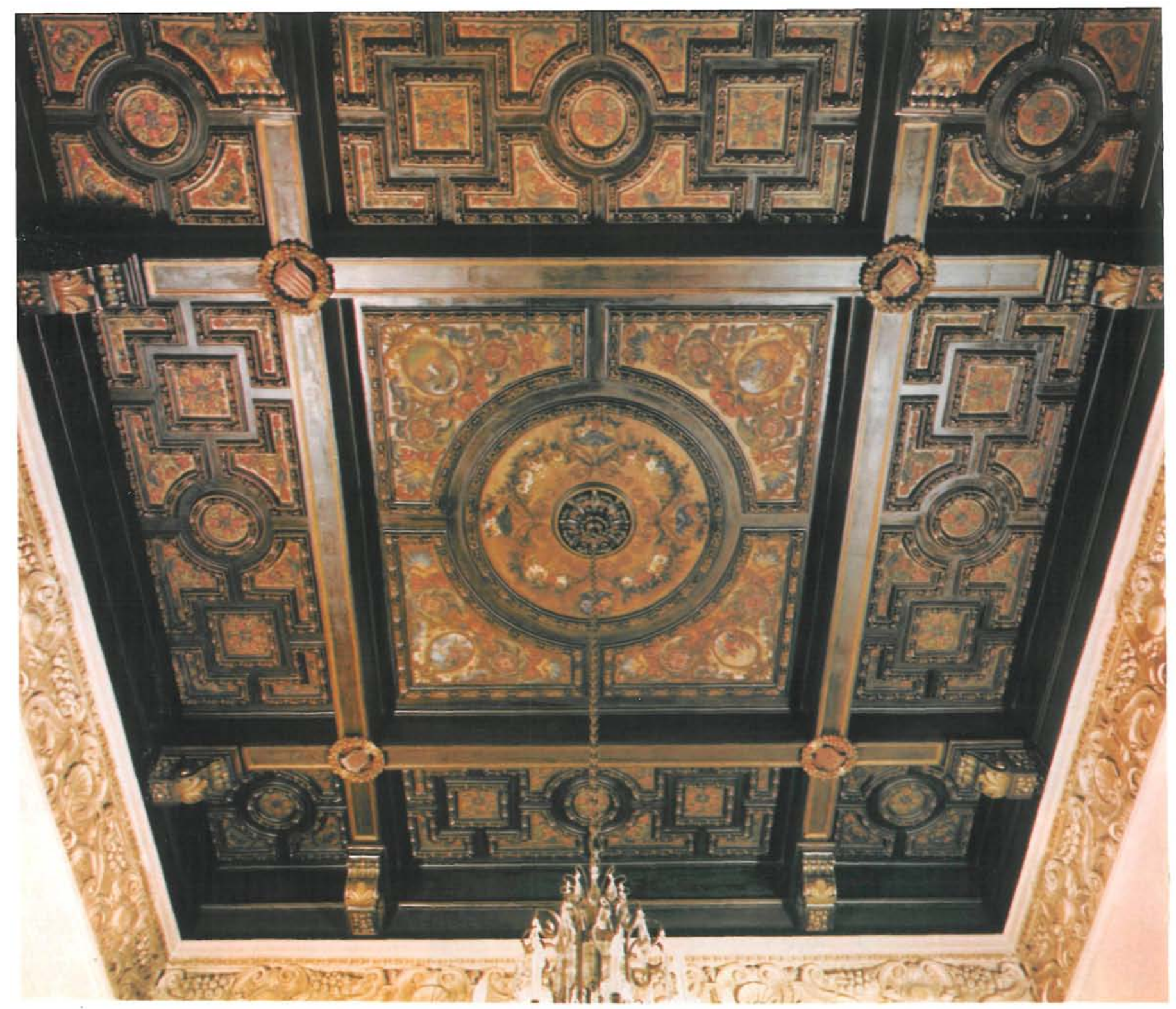

La totalidad de los cuartos de baño han sido remodelados y reconstruidos revistiéndolos de mármol y azulejos del tipo y características anteriores, realizados por la artesania sevillana. Los pertenecientes a suites y zonas nobles han sido tratados totalmente en mármol.

Destaca también la realización de una gran suite con un salón de más de $100 \mathrm{~m}^{2}$ y tres dormitorios y servicios complementarios. Es, en su conjunto, una pieza importante que ha sustituido a la primitiva, de menores proporciones.

Comenzada la obra se puso de manifiesto la complejidad de la misma, dada la peculiar estructura del edificio y su mal estado de conservación derivado de una construcción original de baja calidad. Así, por ejemplo, la tabiqueria estaba prácticamente sustentada por los guarnecidos de yeso, lo que al iniciar la restauración ocasionó su desplome, dado que el material de anclaje primitivo era prácticamente inexistente. Situación análoga presentaba el revoco de la fachada que, si bien inicialmente ofrecia un aspecto aparentemente normal, al iniciarse su restauración se observó la mala calidad del material de agarre de los ladrillos, lo que obligó a realizar un enfoscado profundo previo, a fin de poder realizar un nuevo revoco con garantia de resistencia para el futuro.

Mención especial merece el mal estado que presentaba la estructura de cubierta, que ha obligado a un importante tratamiento de consolidación.

En general, toda la carpinteria interior se ha utilizado nuevamente después de la oportuna restauración. De la carpinteria exterior, ha habido necesidad de renovar toda la correspondiente a la fachada oeste, muy castigada por el sol; la del resto del Hotel ha requerido numerosas reparaciones y restauraciones para mantenerla en un adecuado nivel de uso. 

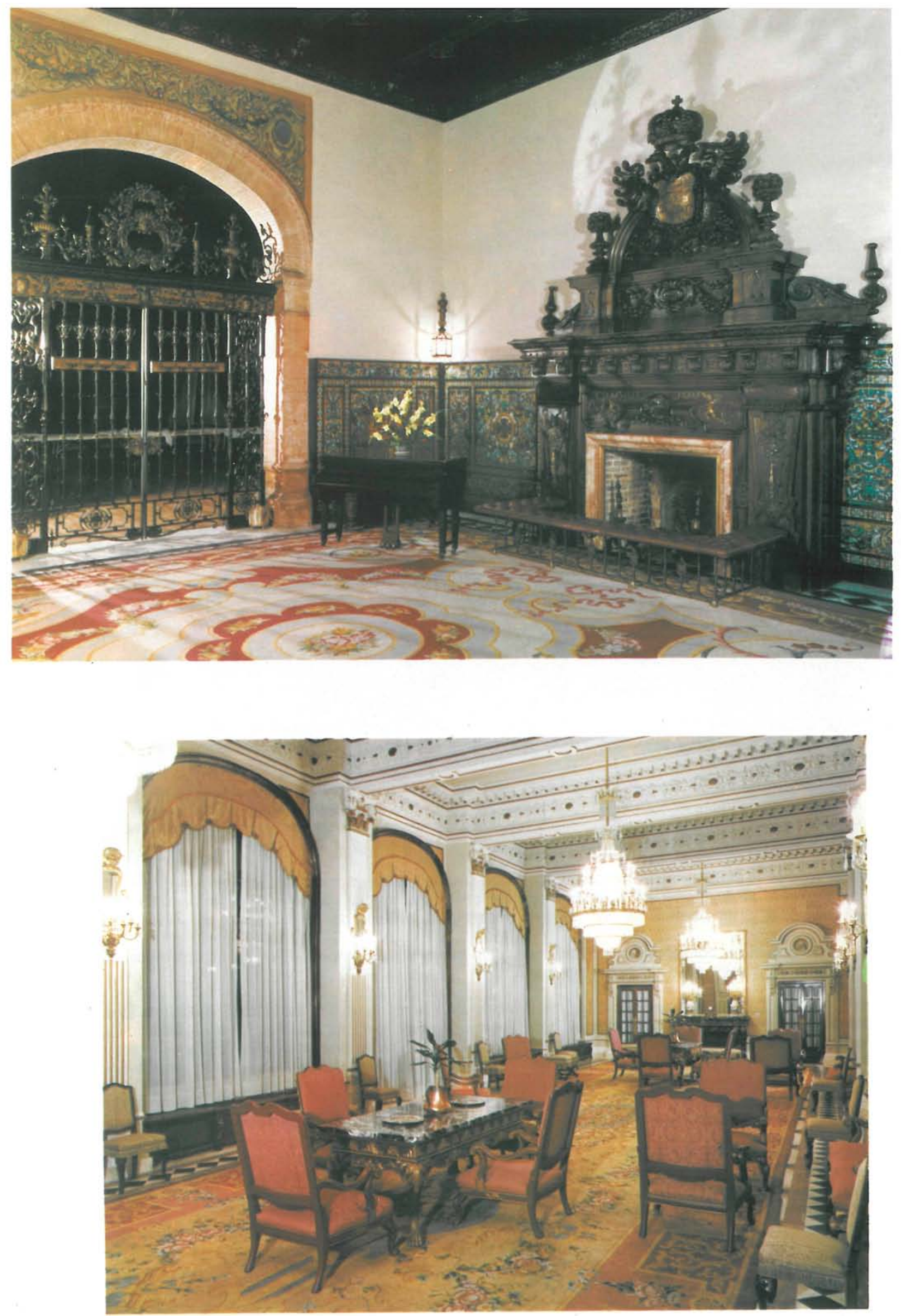


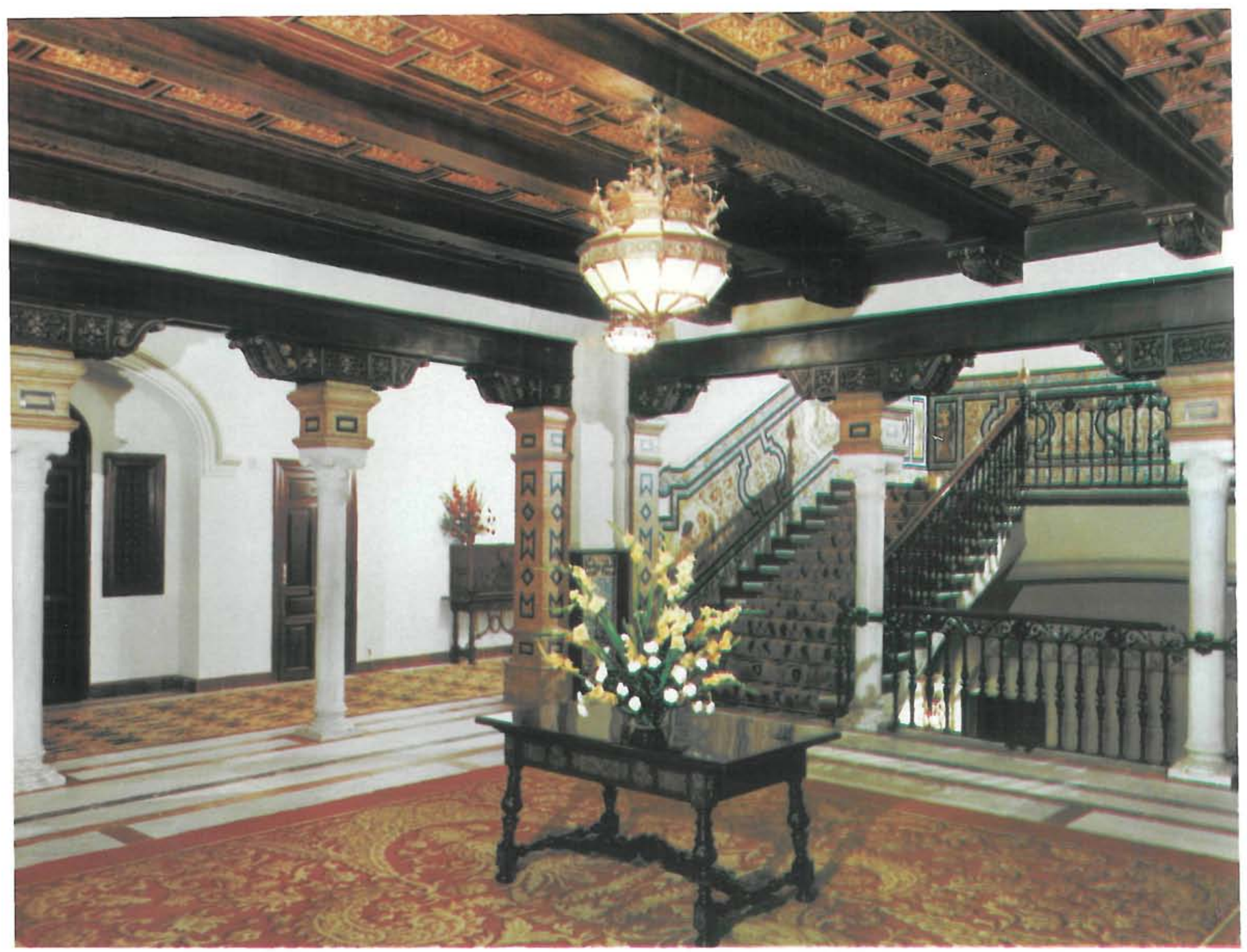

Importante novedad ha sido la restauración de toda la verja del jardín, asi como la instalación de una nueva en la fachada de la calle de Maria de Padilla. El montaje de esta verja sobre un nuevo muro de ladrillo con sus correspondientes pilastras, rematadas por farolas, realza la importancia del jardin y en general de todo el edificio.

En el recinto exterior resalta el haber dotado al Hotel de un estacionamiento cubierto y otro exterior con un total de 70 plazas de aparcamiento. La construcción del sótano ofreció dificultades técnicas, dado el nivel freático de Sevilla y la inadecuada calidad de su subsuelo en aquella zona.

Se protegieron y conservaron las especies importantes existentes en el jardin planificando y realizando la reposición de la jardineria menor.

En la planta baja se ha respetado cuidadosamente todo lo monumental, restaurándose la totalidad de los artesonados. Fue asimismo desmontado pieza por pieza, para su traslado, el friso de azulejo de la que hoy es entrada al nuevo restaurante, viéndose en la necesidad de realizar copia de otros dos módulos iguales a los existentes, labor realizada lo mismo que la del hall de entrada, por artesanos sevillanos, con gran rigor y fidelidad.

Los salones principales se han restaurado dentro de su estilo primitivo; se destaca también la labor efectuada en sus artesonados y la limpieza de las paredes. Por encima de todo lo anterior es destacable la restauración y puesta a punto, en cuanto a la instalación eléctrica se refiere, de todas las arañas y apliques de estos salones, trabajo efectuado tras una paciente labor de engarzar la cristaleria, asi como complementar aquellas piezas que el tiempo había perdido y de las cuales no habia material de repuesto alguno.

Capitulo aparte merece el problema surgido en el antiguo comedor de banquetes del Hotel, en el cual, una vez restaurado y ante el mal estado que presentaba su antiguo piso de parquet, se decidió levantarlo, lo que permitió comprobar que no era debido a la propia madera, sino a que la estructura de hormigón amenazaba ruina por estar seccionadas las cabezas de las vigas que sujetaban los 

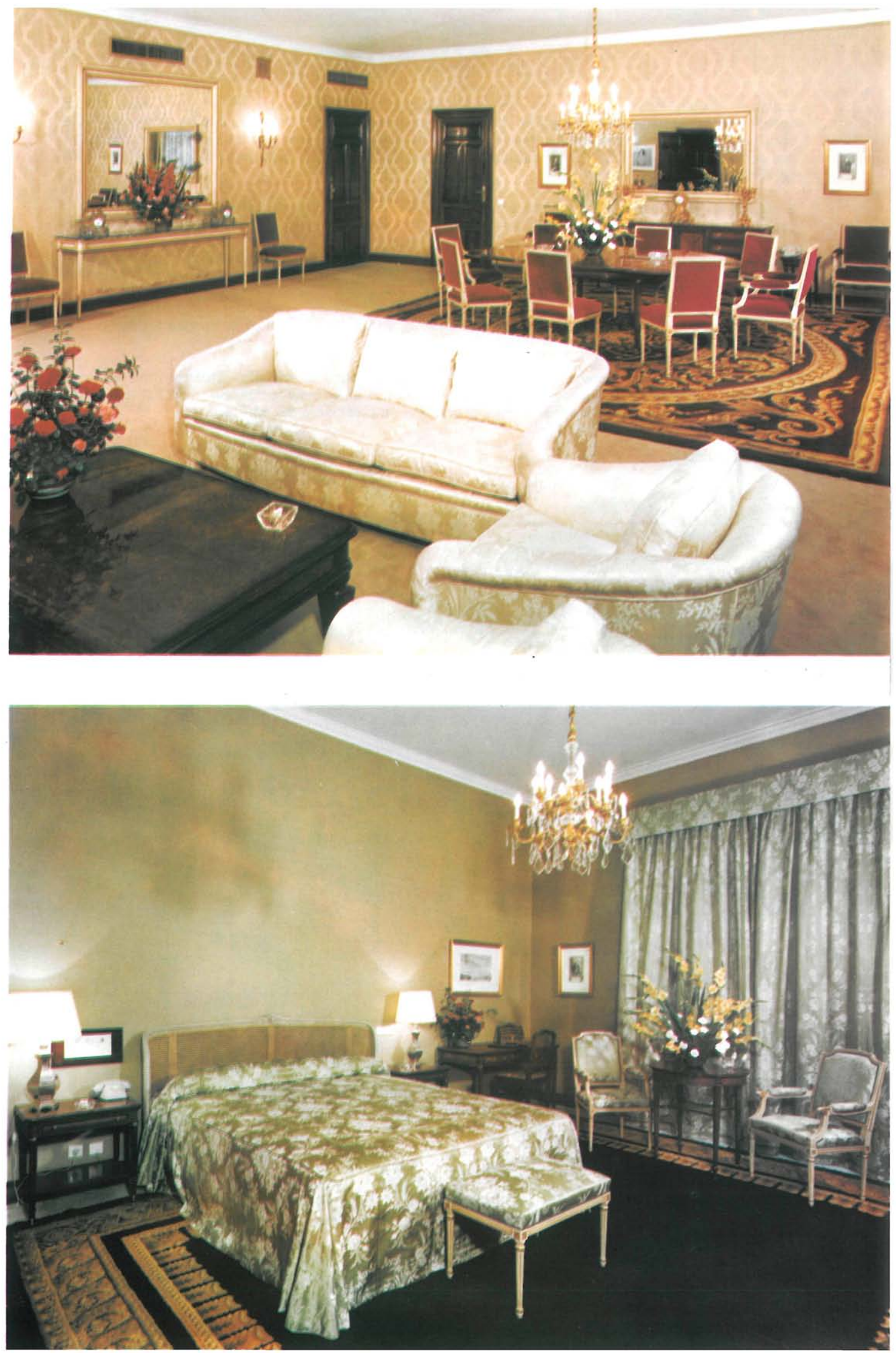
forjados. Fue una obra especialmente delicada arriostrar todo este forjado sin tirarlo; $y$, teniendo en cuenta que las instalaciones generales ya estaban realizadas, hubo momentos que se pensó que esta obra podria retrasar la apertura del Hotel en más de tres meses. Las dificultades fueron superadas por especialistas de la Empresa Constructora, en tan sólo 9 dias, lo que permitió seguir el resto de las fases sin mayores retrasos.

En la galeria principal se ha instalado un bar americano y la cafetería de desayunos, aprovechando el espacio que ocupaban unas antiguas habitaciones cuya única ventilación era esta galeria.

Este planteamiento permite que el público, en general, entre hasta el fondo del Hotel y enlace con el nuevo restaurante situado en la planta de sótano. Lo más importante es haber dado vida a las galerias y al patio central, antes un tanto olvidados.

Hubieron de renovarse todas las bajantes, sin tener que tocar el piso de azulejos que rodean dichas galerias.

El patio hubo de ser desmontado en su totalidad para, después de vaciarlo, situar el gran aljibe de $800 \mathrm{~m}^{3}$ que garantice el abastecimiento instantáneo en cualquier emergencia. Se pudo asi renovar su pavimento e instalar una nueva fuente más acorde con la prestancia dél edificio.

FOTOS: CARTAGUIA

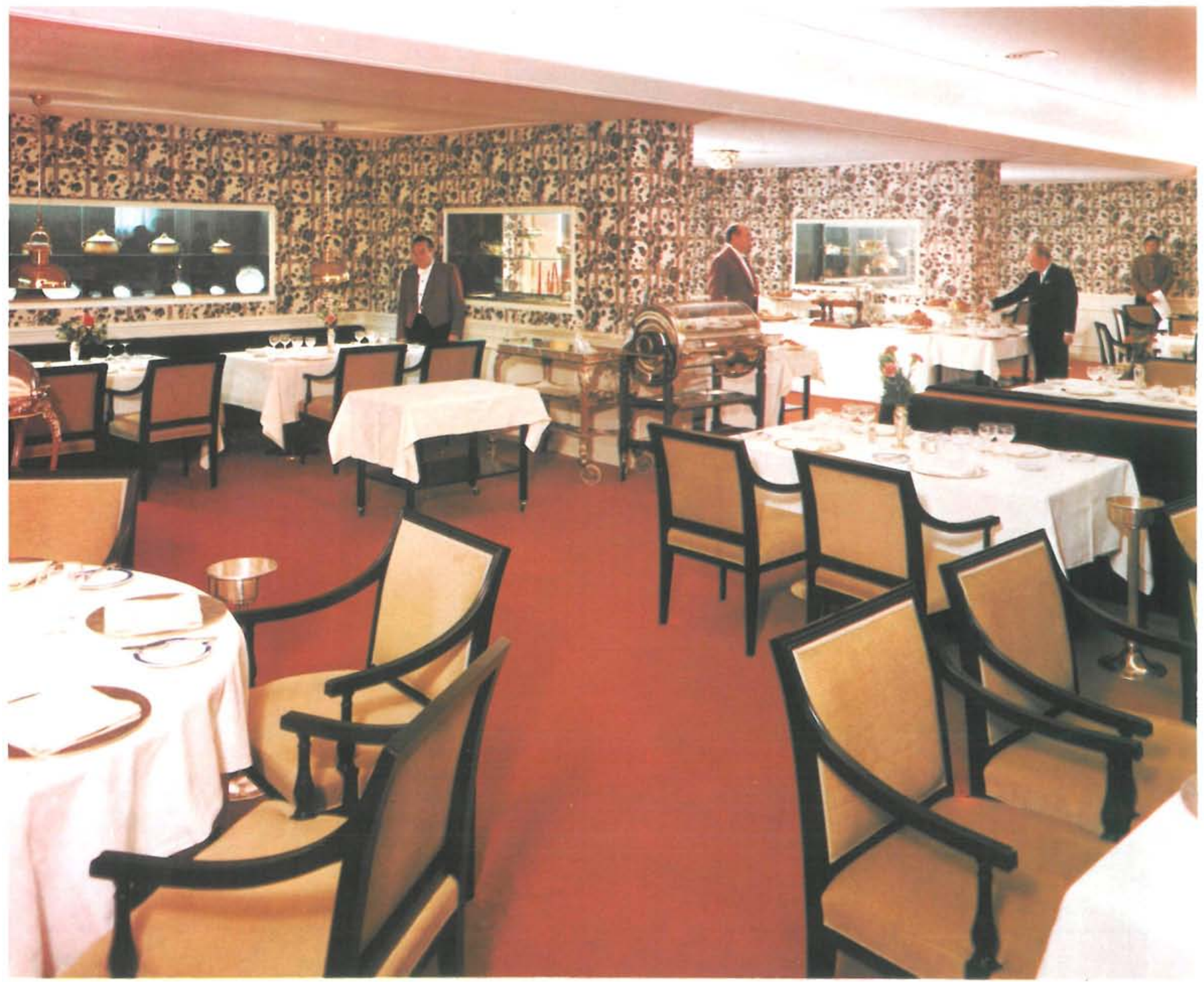


Novedad de la reforma, y de cara a una mejora en la explotación, ha sido la creación de un moderno restaurante de lujo en línea con el ambiente del Hotel. Se situó en el semisótano a fin de lograr que estuviera en la misma planta que la cocina. Esta instalación permitirá dar servicios, tanto a los clientes alojados, como de paso, y ofrece la comodidad de tener acceso no sólo desde el interior del establecimiento, sino también desde el estacionamiento de vehículos.

Los sótanos han sido reestructurados conforme a las exigencias de un Hotel de cinco estrellas instalándose, en los mismos, una moderna lavanderia; nuevas cámaras frigorificas, comedores y vestuarios de personal, economato, talleres, un self-service y una nueva cocina con todos los adelantos que las nuevas secciones de la restauración exigen.

Finalmente, el Hotel Alfonso XIII ha entrado en servicio nuevamente, acorde con las exigencias actuales, tanto de la clientela como de la reglamentación, para poder ser clasificado como Hotel de cinco estrellas, Gran Lujo, categoria sólo alcanzada por siete Hoteles en toda España.

\section{résumé}

TRANSFORMATION ET MODERNISATION DE L'HOTEL ALFONSO XIII DE SEVILLE - ESPAGNE

Carlos Picardo Castellón, Dr. architecte

Dans cet article, l'auteur décrit la transformation soignée de cet hótel -qui fait partie de l'histoire de la ville dès son inauguration en 1926-, d'où la modernisation générale, afin de le transformer en une installation exemplaire, d'une grande beauté, dotée de tous les éléments de confort qu'exige sa catégorie de grand luxe, condition qu'elle partage avec certains hôtels du pays.

Une attention spéciale a été attachée à la consolidation de la toiture et des murs de remplissage, au renouvellement de la menuiserie extérieure, des grilles, des carreaux, des socles, des lambrissages, des lampes, du plancher de la salle des banquets, installations de plomberie, d'électricité d'air conditionné, des sanitaires, etc.

Cette transformation comprend la création d'une suite magnifique et spacieuse, l'aménagement de salles de bains de grande nagement de salles de bains de grande
classe, de jardins, de stationnements couclasse, de jardins, de stationnements coud'une cafeteria, d'un restaurant au sous-sol, de pièces d'eau, etc.

Le goût soigné de l'architecte, le travail hautement spécialisé de l'entreprise de construction et l'apport d'ouvrages des artisans sévillans les plus renommés ont contribuè à un finissage parfait et à un résultat final d'exception.

\section{summary}

REMODELLING OF THE ALFONSO XIII HOTEL IN SEVILLE - SPAIN Carlos Picardo Castellón. Dr. in Architecture

This article describes the careful remodelling work of this hotel - a part of the city's history since it was opened in 1926 - a general improvement aimed at making this facility a model in its class, a beautiful building fitted with all the amenities becoming its high luxury classification, a category shared with very few hotels in the country.

Special attention has been given to the repair of roofs and walls, renewal of exterio fenestration, grills, artistic glazed tiling. dadoes, coffered ceilings, lamps, floor construction in the banquet hall, plumbing, electric system, air conditioned and providing adequate services.

A new magnificent, roomy suite has been incorporated, as well as new classy bathrooms, gardens, roofed carpark, and $800 \mathrm{m3}$ water tank , ro a breakfst coffee room, water tank, a bar, a breaktst coffee room, a cesses from the inside and the outside, fountains, etc. The refined taste of the architect, the highly
specialised work of the contractor and the participation of the qualified Sevillian craftsparticipation of the qualified Sevillian craftsing of the facility and to the highly successful final result.

\section{zusammenfassung}

REFORM UND MODERNISIERUNG DES HOTELS ALFONSO XIII IN SEVILLA, SPANIEN

Carlos Picardo Castellón, Dr. Architekt

In diesem Artikel wird die sorgfältige Reform dieses Hotels - ein Teil der Geschichte dieser Stadt, seit der Eröffnung im Jahre 1926 - mit der folglichen Modernisierung beschrieben, durch welche es in einen beispielshaften $\mathrm{Bau}$ besonderer Schönheit und mit dem für die Kategorie eines Luxus-Hotels erforderlichen Komfort verwandelt werden sollte, eine Voraussetzung, die nur wenige Hotels in diesem Lande erfüllen.

Besondere Beachtung wurde der Verfestigung der Dach und Trennvandbauten geschenkt; Erneuerung der äusseren Schreinerarbeiten, Zäune, Kachein, Sockel, Täfelungen, Lampen, Schmiedearbeiten im Speiseraum mit Bänken, Klempnerei-Installationen, Elektrizität und Klimaanlage; geeignete Einrichtungen usw.

Man erzielte: eine herrliche, geräumige Suite, Badezimmer grosser Kategorie, Gartenanlagen überdachte Parkplätze, eine Zisterne mit einem Fassungsvermögen von $800 \mathrm{~m} 3$, Bar, Cafeteria für die Einnahme des Frühsü̈cks, Cin Restaurant im Souterrain des mit Zugang von aussen und innen-, Springbrunnen.

Der ausgezeichnete Geschmack des Architekten, die hoch spezialisierte Arbeit der Baufirma und die verwendung der reinsten Kunsthandwerksartikel Sevillas haben $z u$ einer einwandfreien Fertigstellung und zu einer einwandreien Fertigstellung und
einem hervorragenden Ergebnis geführt. 